\title{
MiR-130b plays an oncogenic role by repressing PTEN expression in esophageal squamous cell carcinoma cells
}

Tingting $\mathrm{Yu}^{1 \dagger}$, Risheng $\mathrm{CaO}^{1 \dagger}$, Shuo $\mathrm{Li}^{1 \dagger}$, Mingen Fu${ }^{1}$, Lihua Ren ${ }^{1}$, Weixu Chen ${ }^{1}$, Hong Zhu ${ }^{1}$, Qiang Zhann ${ }^{2^{*}}$ and Ruihua Shi ${ }^{1,3^{*}}$

\begin{abstract}
Background: Esophageal carcinoma is one of the most common malignancies with high cancer-related morbidity and mortality worldwide. MicroRNAs (miRNAs) are a class of small non-coding RNAs that regulate a wide variety of cellular processes, and also play an important role in the development and progression of cancers. In a previous microarray study, we demonstrated that miR-130b was upregulated in esophageal squamous cell carcinoma (ESCC) tissues. However, the biologic functions and the molecular mechanism of miR-130b in ESCC remain to be elucidated.
\end{abstract}

Methods: qRT-PCR assays were used to quantify miR-130b expression levels in ESCC samples. Novel targets of miR-130b were identified via a bioinformatics search and confirmed using a dual-luciferase reporter system. Western blotting and qRT-PCR assays were used to quantify the expression of the target gene PTEN (phosphatase and tensin homolog) and the downstream effector, Akt. ESCC cells over- or underexpressing miR-130b were analyzed for in vitro biologic functions.

Results: High levels of miR-130b were identified in 20 ESCC samples following comparison with adjacent non-neoplastic tissues. We confirmed that miR-130b interacted with the $3^{\prime}$-untranslated region of PTEN, and that an increase in the expression level of miR-130b negatively affected the protein level of PTEN. However, the dysregulation of miR-130b had no obvious impact on PTEN mRNA. As Akt is a downstream effector of PTEN, we explored if miR-130b affected Akt expression, and found that miR-130b indirectly regulated the level of phosphorylated Akt, while total Akt protein remained unchanged. Overexpression of miR-130b increased the proliferation of ESCC cells and enhanced their ability to migrate and invade. In contrast, the proliferation, migration, and invasion of ESCC cells were weakened when miR-130b expression was suppressed, which was reversed by PTEN-targeted siRNA.

Conclusion: The results indicate that miR-130b plays an oncogenic role in ESCC cells by repressing PTEN expression and Akt phosphorylation, which would be helpful in developing miRNA-based treatments for ESCC.

Keywords: Esophageal squamous cell carcinoma, miR-130b, Oncogenic, PTEN

\footnotetext{
*Correspondence: zhanq33@163.com; ruihuashi@126.com

${ }^{\dagger}$ Equal contributors

${ }^{2}$ Department of Gastroenterology, Wuxi People's Hospital Affiliated with

Nanjing Medical University, 299 Qingyang Road, Wuxi 214023, China

'Department of Gastroenterology, First Affiliated Hospital of Nanjing Medical

University, 300 Guangzhou Road, Nanjing 210029, China

Full list of author information is available at the end of the article
} 


\section{Background}

Esophageal carcinoma is one of the most common malignancies with high cancer-related morbidity and mortality worldwide. Esophageal squamous cell carcinoma (ESCC) and esophageal adenocarcinoma are the two major histologic types of esophageal carcinoma [1]. The incidence of esophageal carcinoma shows significant regional differences [2]. Notably, the incidence of ESCC is particularly high in the so-called Asian belt, which includes Turkey, northeastern Iran, Kazakhstan, and northern and central China, with more than 100 cases per 100,000 people reported annually [3]. The carcinogenesis of ESCC is proposed as a multistep process. Previous studies have shown that tobacco and alcohol consumption are associated with a higher risk of ESCC [4]. Other risk factors include low socioeconomic status, nutritional deficiencies, excess intake of hot beverages and genetic predisposition [5,6]. Although diagnostic and treatment methods have been developed in recent years, the five-year overall survival of ESCC remains $<14 \%$ [1]. Therefore, more specific and sensitive biomarkers for diagnosis and targeted therapy of ESCC are urgently needed.

MicroRNAs (miRNAs) are a class of small non-coding RNAs that are 17-24 nucleotides in length [7]. It is estimated that more than $50 \%$ of the miRNAs are located in cancer-related regions and fragile sites of chromosomes [8]. Increasing evidence shows that dysregulation of miRNAs leads to the development and progression of cancers [9]. The up- or downregulation of miRNAs is correlated with specific tumor types; different human cancers have distinct miRNA expression profiles [10]. Studies have shown that aberrant expression of miRNAs affects cell proliferation, apoptosis, metastasis, and sensitivity to chemotherapy and radiotherapy in multiple cancers [11]. This highlights the potential for using expression profiles of specific miRNAs for cancer diagnosis or therapy.

MiR-130b, located at the 22q11 locus [12], plays an oncogenic role in gastric, liver, and endometrial cancers [13-15], and acts as a tumor suppressor in ovarian cancer and thyroid papillary carcinoma [16,17]. A recent study revealed a correlation between miR-130b and prognoses of postoperative esophageal cancer patients, and suggested that upregulation of miR-130b might be an unfavorable factor for ESCC patients [18]. However, the expression levels and the functions of miR-130b in ESCC have not been reported so far. In the present study, we explored the regulation of miRNAs that may be involved in the development and progression of ESCC. We analyzed miRNA expression profiles in matched primary ESCC and corresponding normal esophageal tissues via miRNA microarray analysis and quantitative real-time PCR (qRT-PCR). A dual-luciferase reporter assay was used to verify that phosphatase and tensin homolog (PTEN) is a downstream target of miR-130b. Functional analyses regarding cell proliferation, migration, and invasion were performed to investigate the effect of miR-130b expression on ESCC cells in vitro.

\section{Methods}

\section{Cell lines and clinical samples}

Matched ESCC and corresponding adjacent normal esophageal tissues were obtained from 23 patients who underwent esophagectomy for primary ESCC in the First Affiliated Hospital of Nanjing Medical University (Table 1). None of the patients had received preoperative chemotherapy or radiotherapy. All tissue samples were frozen in liquid nitrogen immediately after resection and stored at $-80^{\circ} \mathrm{C}$. The histologic diagnosis of these tissue samples was confirmed by a pathologist. This study was approved by the Committee for Ethical Review of Research at the First Affiliated Hospital of Nanjing Medical University, and informed consent was obtained from all the patients involved. The human ESCC cell lines, Eca109 and TE13, were purchased from the Cell Bank of the Chinese Academy of Sciences (Shanghai, China).

\section{Cell culture and transfection}

Eca109 and TE13 cell lines were cultured in Roswell Park Memorial Institute (RPMI)-1640 or Dulbecco's modified eagle media (Wisent Inc., Quebec, Canada) supplemented with $10 \%$ fetal bovine serum (Wisent Inc.) and 1\% penicillin-streptomycin (Invitrogen of Thermo Fisher Scientific Inc., Waltham, MA, USA) in a humidified $5 \% \mathrm{CO}_{2}$ atmosphere at $37^{\circ} \mathrm{C}$. The miR-130b mimic (miR$130 \mathrm{bm}$ ), negative control of miRNA mimic (NC), miR-130b inhibitor (miR-130bi), negative control of miRNA inhibitor (iNC) and PTEN-targeted small interfering RNA (siPTEN)

\section{Table 1 Clinical features of 3 ESCC patients for subjection of Agilent microarray analysis and 20 patients for qRT-PCR validation}

\begin{tabular}{lll}
\hline Clinical parameters & Screening set $(\mathbf{n}=\mathbf{3})$ & Validation set $(\mathbf{n}=\mathbf{2 0})$ \\
\hline Age (years) & 2 & 8 \\
$\leq 60$ & 1 & 12 \\
$>60$ & & \\
Gender & 2 & 14 \\
Male & 1 & 6 \\
Female & & \\
TNM staging & 2 & 13 \\
II & 1 & 7 \\
III & & \\
Tumor diameter & 3 & 16 \\
$\leq 5 \mathrm{~cm}$ & 0 & 4 \\
$>5 \mathrm{~cm}$ &
\end{tabular}


were purchased from GenePharma (Shanghai, China) and transfected at a final concentration of $50-100 \mathrm{nM}$ with Lipofectamine 2000 (Invitrogen) according to the manufacturer's instructions. After $6 \mathrm{~h}$, the cells were returned to normal medium and cultured for an additional 48 (for RNA isolation) or $72 \mathrm{~h}$ (for protein extraction). The inhibition efficiency of siPTEN was also determined (Additional file 1).

\section{MiRNA microarray analysis}

Total RNA was isolated from matched ESCC and corresponding normal esophageal epithelium tissues $(n=3)$ with TRIzol (Invitrogen) according to the manufacturer's instructions. The RNA concentration was measured on a Nanodrop 2000 spectrophotometer (Thermo Fisher Scientific), and the quality of the RNA was examined on a denaturing formaldehyde gel. The RNA was purified (mirVana miRNA Isolation Kit; Ambion of Thermo Fisher Scientific), and $200 \mathrm{ng}$ from each tissue sample was labeled and hybridized (miRNA Complete Labeling and Hyb Kit; Agilent Technologies, Santa Clara, CA, USA) on a miRNA microarray chip (G4870A; Agilent Technologies) encompassing 1205 human and 142 viral miRNA probes. The data were transformed from the hybridization picture with Feature Extraction software v10.7 and integrated using GeneSpring software (Agilent Technologies).

\section{qRT-PCR}

RNA was isolated from ESCC tissue samples and cells as described above and cDNA was synthesized using a TaqMan microRNA Reverse Transcription Kit (Applied Biosystems of Thermo Fisher Scientific) according to the manufacturer's instructions. The expression level of mature miR-130b in the tissues or the ESCC cells was confirmed by a TaqMan microRNA assay (Applied Biosystems). Levels of PTEN mRNA in ESCC cells were quantified using SYBR Green real-time PCR master mix (Applied Biosystems) and specific primers: PTEN [Genebank: NM_000314], 5' -TTTGAAGACCATAACCCACCAC-3' (forward), 5' ATTACACCAGTTCGTCCCTTTC-3' (reverse); and glyceraldehyde-3-phosphate dehydrogenase (GAPDH) [Genebank: NM_002046], 5'-AGCCTCAAGATCAT CAGCAATG-3' (forward), 5'-TGTGGTCATGAGTC CTTCCACG-3' (reverse). The relative expression levels of mature miR-130b and PTEN mRNA were calculated by the $2^{-\Delta \Delta \mathrm{Ct}}$ method and normalized to U6 snRNA and GAPDH mRNA levels, respectively. All PCR reactions were performed on a StepOne Plus RT-PCR instrument (Applied Biosystems).

\section{Dual-luciferase reporter assay}

A 59 bp fragment from the 3 '-untranslated region (UTR) of PTEN containing the putative binding sequences for miR-130b was synthetized cloned into the firefly luciferase
pGL3-control vector (Invitrogen). For the reporter assay, Eca109 cells (in logarithmic growth phase) were plated in a 24-well culture plate at a density of 8000 cells/well. The cells in each well were co-transfected with $25 \mathrm{nM}$ of miR-130bm, miR-130bi or NC, $800 \mathrm{ng} / \mu \mathrm{L}$ of miR-130bpGL3 vector and $0.8 \mathrm{ng} / \mu \mathrm{L}$ of pRL-TK vector (Invitrogen) using Lipofectamine 2000 according to the manufacturer's instructions. The cell lysates were collected $24 \mathrm{~h}$ after transfection. The firefly and Renilla luciferase activities were measured using the dual-luciferase reporter assay system (Promega Corp., Madison, WI, USA). The luciferase activity was detected on a GLOMAX20/20 luminometer (Promega Corp.) and normalized to the Renilla luciferase activity.

\section{Western blot analysis}

Proteins were extracted from harvested Eca109 and TE13 cells using radioimmunoprecipitation assay buffer (Beyotime, Beijing, China) according to the manufacturer's instructions. Equal amounts of protein lysates $(30 \mu \mathrm{g})$ were separated by sodium dodecyl sulfate-polyacrylamide gel electrophoresis (SDS-PAGE) on a 10\% gel and transferred to a nitrocellulose membrane. The membranes were blocked with $5 \%$ non-fat milk in TBST $(10 \mathrm{mM}$ Tris- $\mathrm{HCl}$ [pH 8.0], $150 \mathrm{mM} \mathrm{NaCl}$, and 0.05\% Tween-20) for $1 \mathrm{~h}$ at room temperature, and incubated overnight with agitation at $4^{\circ} \mathrm{C}$ with primary antibodies against human PTEN, Akt, p-Akt (1:1000; Cell Signaling Technology Inc., Danvers, MA, USA), and GAPDH (1:6000; Bioworld, Visalia, CA, USA). The membranes were washed and incubated with goat anti-rabbit IgG secondary antibody (1:5000; Santa Cruz Biotechnology, Dallas, TX, USA) for $1 \mathrm{~h}$. The immunoreactivity was assessed on a gel-imaging analyzer (Bio-Rad Laboratories Inc., Hercules, CA, USA). The band densities of PTEN, Akt and p-Akt were measured using Image Lab software (Bio-Rad Laboratories, Inc.) and normalized to GAPDH.

\section{Cell proliferation assay}

The transfected cells that were in the logarithmic growth phase were seeded into 96-well plates at a cell density of 2000 cells/well for Eca109 and 5000 cells/well for TE13. Five wells were set up for each transfected group. Cell counting kit- 8 (CCK-8) solution $(10 \mu \mathrm{L})$ was added to one well in each group every $24 \mathrm{~h}$ for four consecutive days, incubated at $37^{\circ} \mathrm{C}$ for $2 \mathrm{~h}$, and the optical density was measured at $450 \mathrm{~nm}$ on a microplate reader (Thermo Fisher Scientific Inc.). The proliferation curve was determined using the average optical density values of each group.

\section{Colony formation assay}

After transfection, approximately 300 Eca109 cells were added to each well of 6-well plates and incubated at 
$37^{\circ} \mathrm{C}$ for $2 \mathrm{wk}$. The culture medium was removed twice a week and replaced with fresh medium. On day 14, the cells were washed twice with PBS, fixed with methanol for $30 \mathrm{~min}$ and stained with crystal violet staining solution (Beyotime, Beijing, China) for $30 \mathrm{~min}$. The dishes were washed with PBS three times and air-dried. The colonies that contained $>50$ cells were counted and the colony forming efficiency was calculated.

\section{Anchorage-independent growth ability assay in soft agar}

Two thousand Eca109 cells were trypsinized and suspended in $2 \mathrm{~mL}$ complete medium plus $0.3 \%$ agar (Becton, Dickinson and Co., Franklin Lakes, NJ, USA). The agar-cell mixture was plated on top of a bottom layer with $0.6 \%$ complete medium agar mixture. After $2 \mathrm{wk}$, viable colonies that contained $\geq 50$ cells or were $>50 \mu \mathrm{m}$ in diameter were counted.

\section{Transwell migration and invasion assays}

For the cell migration assay, Transwell chambers (EMD Millipore, Billerica, MA, USA) with 8.0- $\mu \mathrm{m}$-pore-size basement membranes were placed into a 24-well culture plate. For the cell invasion assay, the upper surface of the membrane was evenly covered with $50 \mu \mathrm{L}$ of $1 \mathrm{mg} / \mathrm{mL}$ Matrigel (Becton, Dickinson and Co.). Briefly, $200 \mu \mathrm{L}$ serum-free cell suspension containing $1 \times 10^{5}$ Eca109 cells was added to the upper compartment of the chamber, and the lower compartment was filled with RPMI-1640 medium containing $10 \%$ fetal bovine serum as a chemoattractant. After incubation at $37^{\circ} \mathrm{C}$ for $48 \mathrm{~h}$, the cells were fixed with $75 \%$ ethanol and stained with crystal violet staining solution. Non-migrating cells on the upper surface of the membrane were removed with cotton swabs. The migratory and invasive cells in each chamber were counted from at least five randomly chosen high power fields.

\section{Statistical analysis}

Statistical analyses were performed using SPSS 17.0 software (SPSS Inc., Chicago, IL, USA). The expression of miR-130b in ESCC and matched normal esophageal tissues was analyzed by paired-samples $t$-test, and independent-samples $t$-tests were used for other data analyses; $P<0.05$ was considered statistically significant. All data from this study were obtained from at least three independent experiments and are expressed as mean \pm SEM.

\section{Results}

\section{MiR-130b is overexpressed in ESCC tissues}

To determine the effect of miRNAs on the development and progression of ESCC, we first performed a miRNA microarray analysis (cutoff, $>2.0$ fold-change). The results of the microarray analysis, which had been deposited to Gene Expression Omnibus (GEO) database [GEO: GSE59973], showed that 51 miRNAs were significantly overexpressed in ESCC tissues when compared with normal esophageal tissues, including miR-130b, which showed a fivefold-change over the normal esophageal tissues (Table 2). Quantification of miR-130b expression in 20 ESCC and matched normal esophageal tissue samples confirmed the microarray results (Figure 1).

\section{PTEN is a target of miR-130b}

By integrating the search results of TargetScan and miRDB programs (Figure 2A), we identified the tumor suppressor gene PTEN as a potential target of miR-130b. A dualluciferase reporter assay was performed to verify that the 3 '-UTR of PTEN mRNA is a direct target of miR-130b. The luciferase activity of Eca109 cells transfected with miR-130bm was reduced by approximately $60 \%$ when compared to the $\mathrm{NC}$ transfected cells, whereas miR-130bi increased the activity (Figure 2B).

\section{MiR-130b regulates expression of PTEN and phosphorylation of Akt}

To investigate the possible underlying molecular mechanism of miR-130b in ESCC tissues, Eca109 and TE13 cells were transfected with miR-130bm and miR-130bi to increase and decrease miR-130b expression, respectively. PTEN targeted siRNA was also co-transfected with miR-130bi to identify the effect of PTEN protein expression. Western blotting (Figure 3A-D) and qRT-PCR (Figure 3G, H) analyses showed that miR-130b negatively regulated levels of PTEN protein but not mRNA, indicating regulation at the translational level.

PTEN is the main negative regulator of the phosphatidylinositol 3 kinase/Akt pathway [19]. Therefore, we explored whether miR-130b regulation of PTEN protein levels affects Akt phosphorylation. Western blotting showed that upregulation of miR-130b was accompanied by an increase in the levels of phosphorylated Akt (p-Akt) (Figure 3A,B,E,F). Conversely, p-Akt levels decreased when the expression of miR-130b was suppressed.

Table 2 The miRNA microarray data of highly upregulated (fold-change $>5.0$ ) miRNAs in esophageal squamous cell carcinoma (ESCC) tissues compared with normal esophageal tissues

\begin{tabular}{lll}
\hline Up-regulated miRNAs & Fold-change & $\boldsymbol{P}$ \\
\hline miR-7 & 10.91 & 0.005 \\
miR-196b & 7.67 & 0.030 \\
miR-424 & 6.75 & 0.008 \\
miR-18b & 6.67 & 0.002 \\
miR-223 & 6.31 & 0.003 \\
miR-130b & 5.18 & 0.005 \\
\hline
\end{tabular}




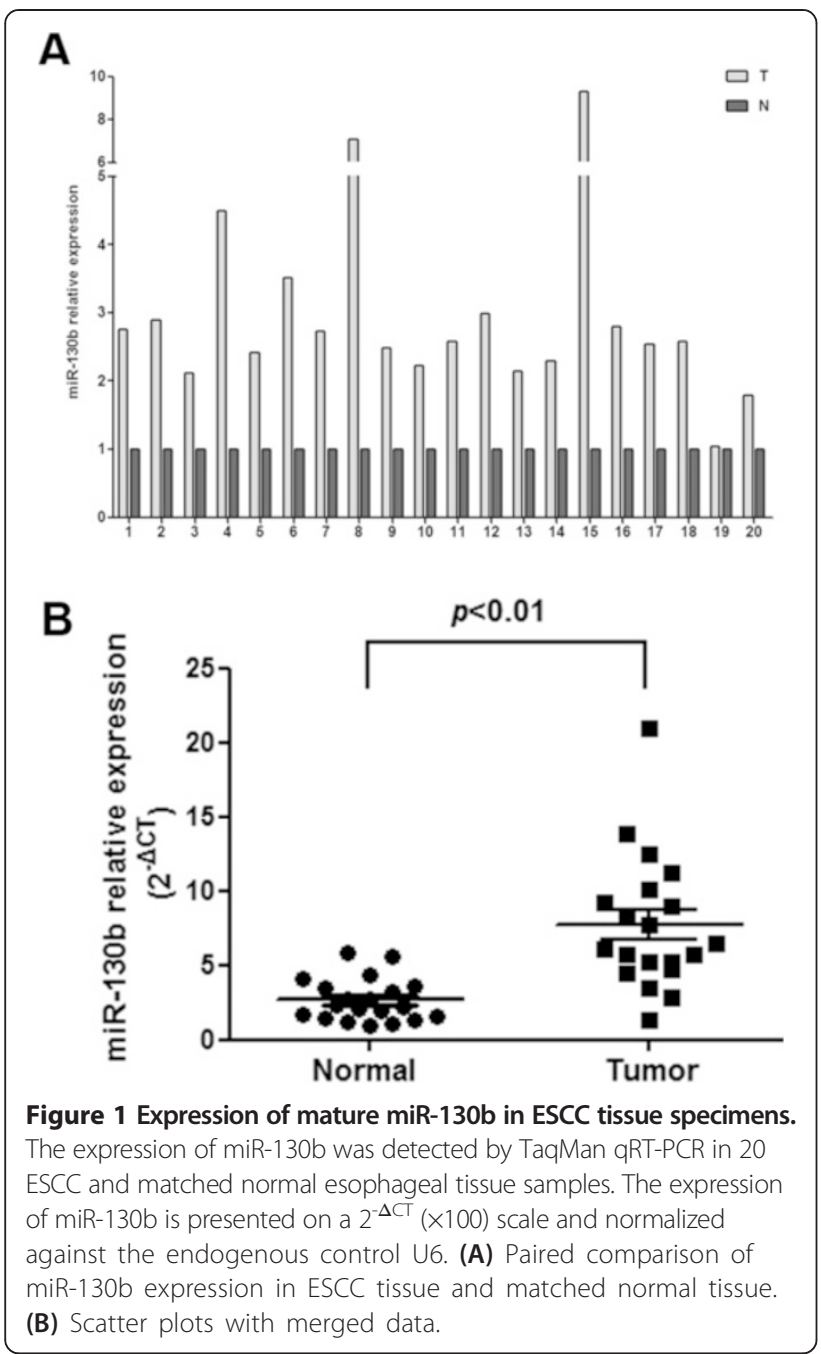

Interestingly, total Akt protein levels remained unchanged, indicating that miR-130b regulates the phosphorylation of Akt, rather than the protein expression.

\section{MiR-130b regulates the proliferation of ESCC cells}

We used CCK- 8 and colony formation assays to examine the proliferative ability of ESCC cells. Cell growth curves were constructed from CCK-8 absorbances in Eca109 and TE13 cells at 24, 48, 72 and $96 \mathrm{~h}$, showing that the rate of cell growth in the miR-130bm transfected group was significantly increased compared with $\mathrm{NC}$ groups $(P<0.05)$ (Figure 4A-D). In contrast, the viability of ESCC cells was inhibited following transfection with miR-130bi, which was reversed by co-transfection with a PTEN-targeted small interfering RNA. Similar results were obtained from colony formation and soft-agar assays in Eca109 cells (Figure 4E-H).

MiR-130b promotes ESCC cell migration and invasion in vitro Tumor cell migration and invasion are one of the leading causes of tumor metastasis. Therefore, we addressed

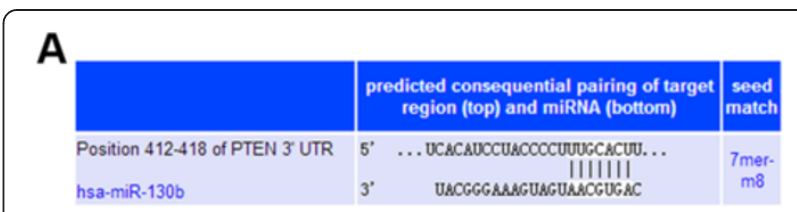

B

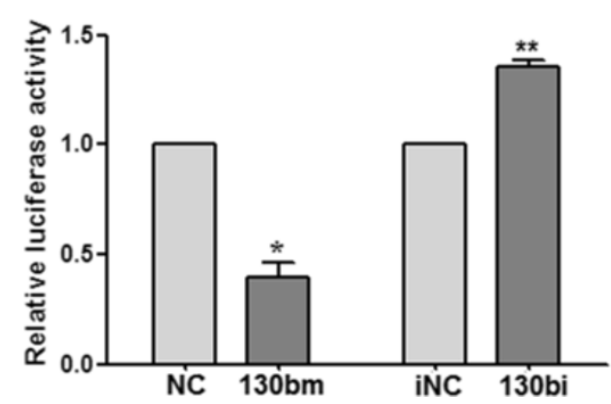

Figure 2 Identification of PTEN as target of miR-130b. (A) Bioinformatic prediction by TargetScan indicated that PTEN mRNA contains a putative miR-130b binding region, located at 412-418 nt of 3'-UTR. (B) A dual-luciferase reporter assay was used to confirm the interaction of miR-130b with PTEN. A fragment of miR-130b that was predicted to bind to the PTEN 3'-UTR was cloned into firefly luciferase pGL3-control vector. The Renilla luciferase plasmid was co-transfected for normalizing luciferase activity. ${ }^{*} P<0.05,{ }^{* *} P<0.01 \mathrm{vs}$. corresponding controls.

whether miR-130b affects the ability of Eca109 cells to migrate and invade. Overexpression of miR-130b significantly increased the number of cells capable of migration and invasion $(P<0.05)$ (Figure 5). Moreover, inhibition of PTEN expression rescued the decreased cell migration and invasion capacities induced by miR-130bi.

\section{Discussion}

A better understanding of the mechanisms underlying the carcinogenesis of ESCC is important for developing more effective diagnostic and therapeutic strategies. As studies on ESCC advanced, they were no longer limited to oncogenes or tumor suppressor genes, and extended to include entire signaling pathways and gene interaction networks. MiRNAs affect a variety of cellular pathways, and a single miRNA can target multiple genes [20,21]. This specific characteristic of miRNAs has made them a topic of intense research in recent years.

It is estimated that miRNAs regulate the expression of $30-60 \%$ of human genes $[20,22]$. MiRNAs regulate gene expression at the transcriptional or translational level by binding to the 3'-UTR of mRNAs [23]. Whether a miRNA acts as a tumor promoter or repressor depends on the gene that it interacts with [24]. Previous studies have reported associations of miR-130b with some types of solid tumors. For example, miR-130b is significantly overexpressed in gastric cancers, which increases cell viability and decreases the expression of runt related transcription factor 3 gene and Bim during transforming growth factor 
A

p-Akt

Akt

PTEN

GAPDH

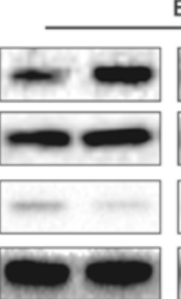

ca109

NC $130 \mathrm{bm}$ iNC 130bi $\begin{array}{r}130 \mathrm{bi}+ \\ \text { siPTEN }\end{array}$

C

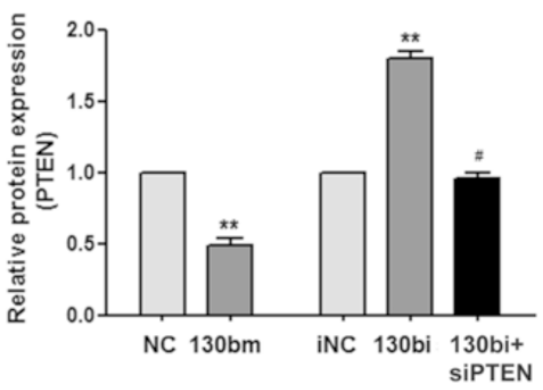

E

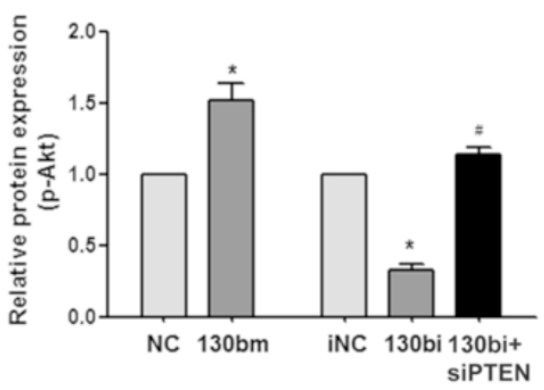

G

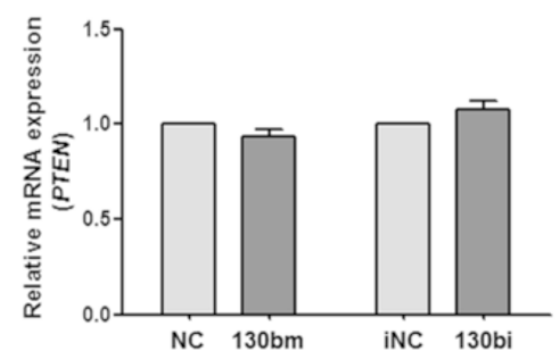

B

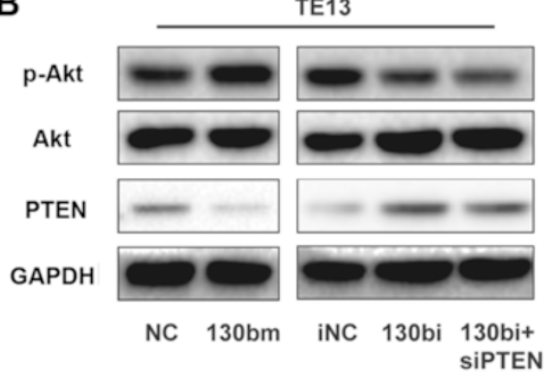

D

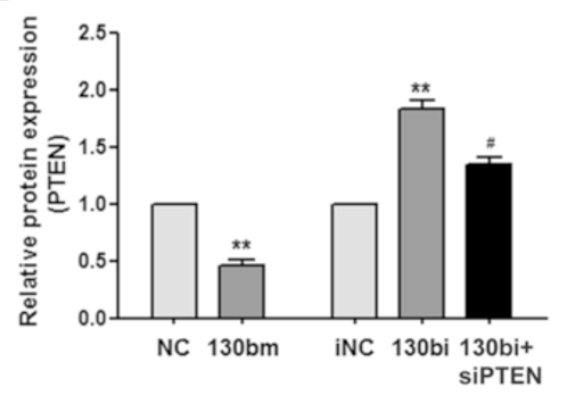

F

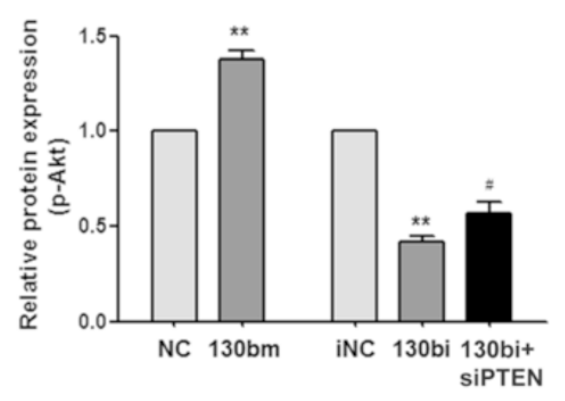

H

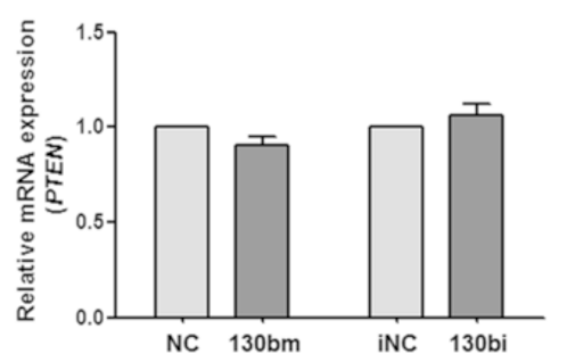

Figure 3 MiR-130b regulates the expression of PTEN and phosphorylation of Akt. Eca109 and TE13 cells were transfected with miR-130b mimic (130bm group) or inhibitor (130bi group); siPTEN was also co-transfected with the miR-130b inhibitor to decrease PTEN expression (130bi + siPTEN group). Protein expression of PTEN, p-Akt and total Akt in Eca109 (A, C, E) and TE13 cells (B, D, F) was determined by Western blot analysis. PTEN mRNA expression was detected by SYBR Green real-time PCR at $24 \mathrm{~h}$ following transfection of Eca109 (G) and TE13 (H) cell lines. The values were normalized to the corresponding controls. GAPDH was the endogenous control. ${ }^{*} P<0.05$, ${ }^{*} P<0.01$ vs. corresponding controls; $\# P<0.05$ vs. 130bi group.

$\beta$-mediated apoptosis [13]. Another study showed that miR-130b promotes $\mathrm{CD}_{133^{+}}$liver tumor-initiated cell growth and self-renewal in vitro and in vivo via tumor protein 53-induced nuclear protein 1 [15]. The expression of miR-130b is increased in hyperplastic endometrium and increases even further in endometrial cancer, which, along with DICER1 dysfunction, leads to tumor aggression both in vitro and in vivo [14]. In contrast, miR-130b is downregulated in ovarian cancer and papillary thyroid carcinoma, and its expression is inversely correlated with the 
A

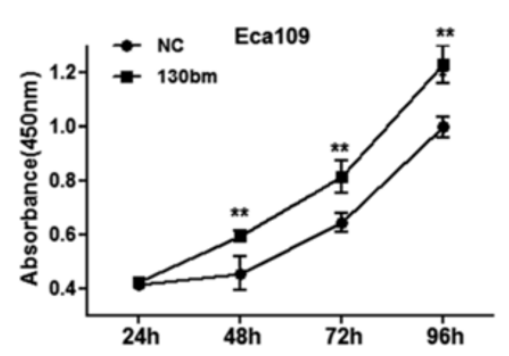

C

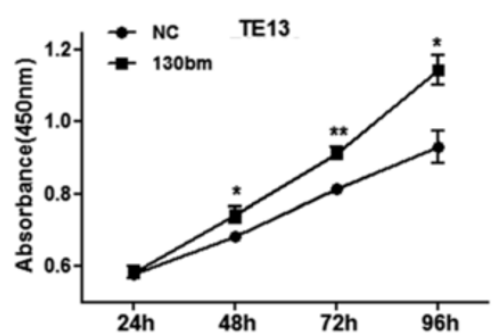

E

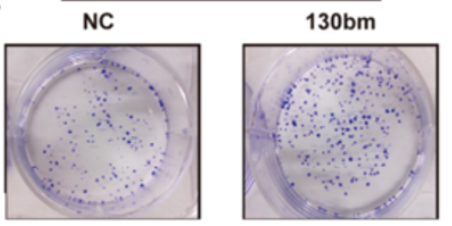

$\mathbf{F}$
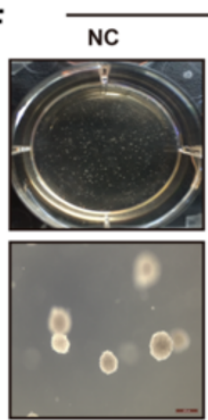

G
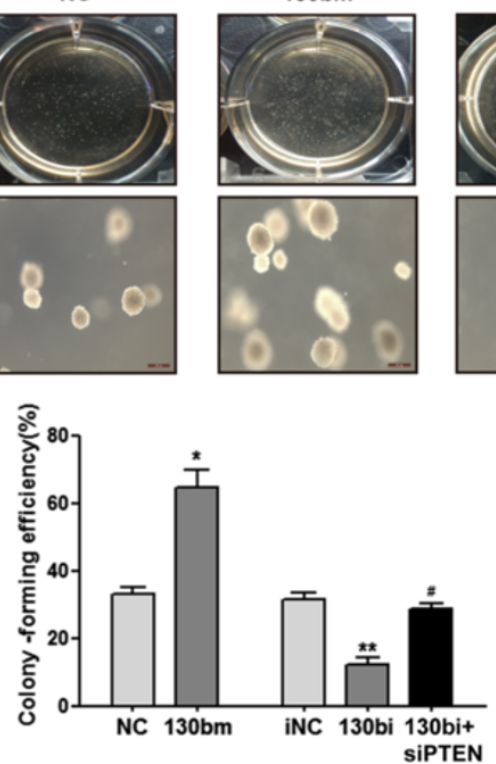

B

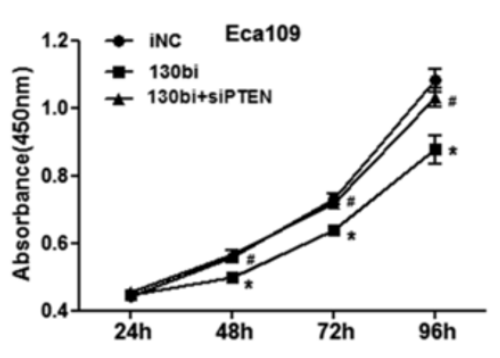

D
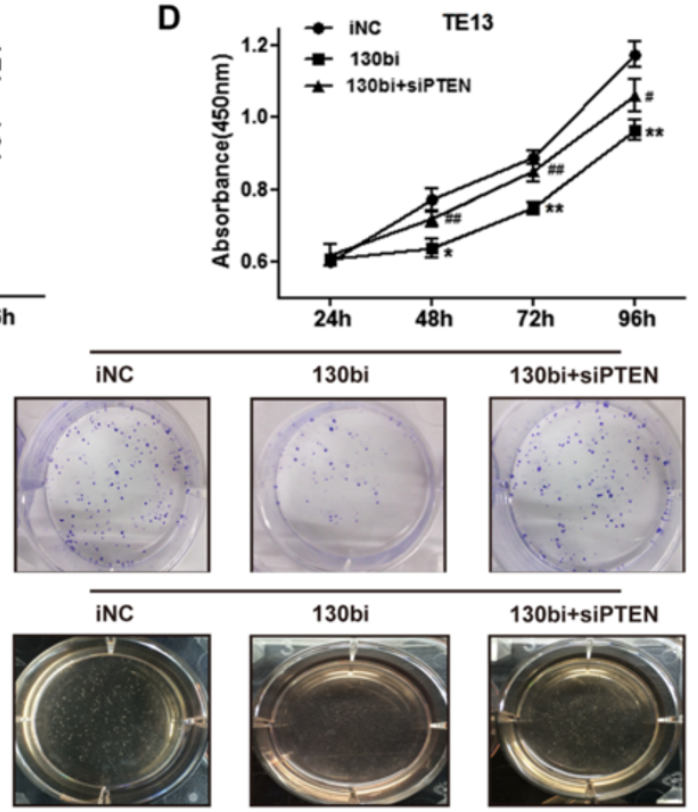

$130 \mathrm{bi}+$ siPTEN
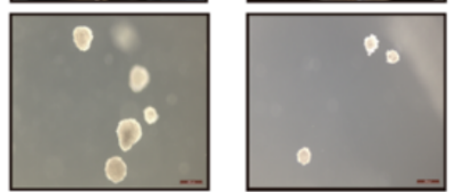

$\mathrm{H}_{-}$

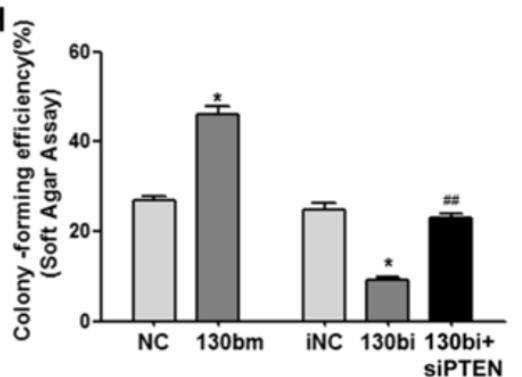

Figure 4 Effect of miR-130b on the proliferation of ESCC cells in vitro. The viability of Eca109 and TE13 cells transfected with miR-130b mimic, negative control, miR-130b inhibitor, inhibitor negative control or siPTEN was detected by CCK-8 at $24,48,72$ and 96 h. The absorbance was measured at $450 \mathrm{~nm}$. (A, B) Proliferation curves of Eca109 cells. (C, D) Proliferation curves of TE13 cells. For colony formation (E, G) and soft-agar $(\mathbf{F}, \mathbf{H})$ assays in Eca109 cells, the following calculation was performed: colony-forming efficiency (\%)=the number of colonies (>50 cells or larger than $50 \mu \mathrm{m}$ )/the number of cells plated per well. The photographs were captured from digital camera or microscope at $40 \times$ magnification, in which the size bar was representative of $200 \mu \mathrm{m}$. The results are expressed as mean \pm SEM of three independent experiments. ${ }^{*} P<0.05,{ }^{*} P<0.01$ vs. corresponding controls; $\# P<0.05$, \#\#P<0.01 vs. 130bi group.

tumor aggressiveness and multidrug resistance in these cancers $[16,17]$.

Recently, the first research report showing a correlation between miR-130b and ESCC in a study comparing ESCC patients with the same tumor node metastasis stage but different prognoses [18]. Their results demonstrated that miR-130b was significantly downregulated in the paracancerous normal esophageal mucous membranes of patients 

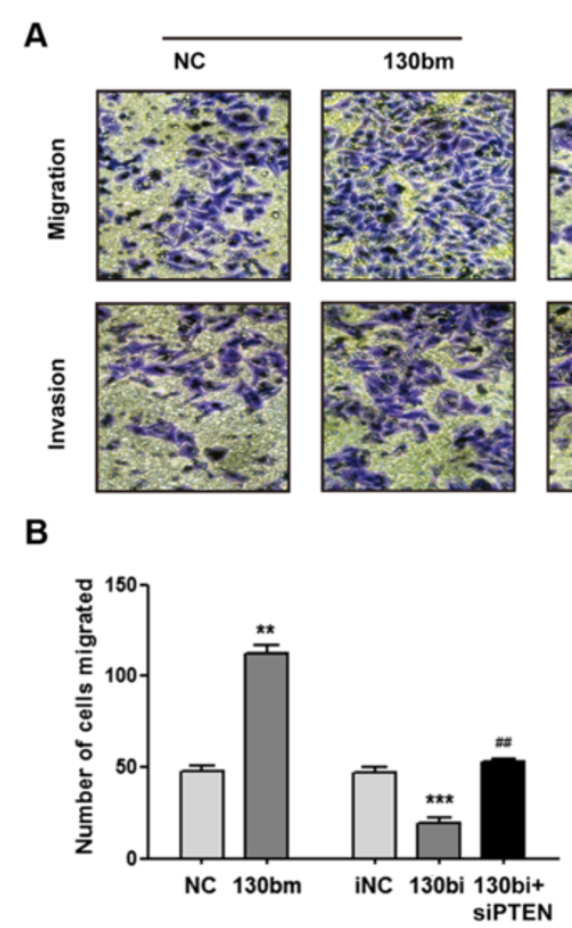

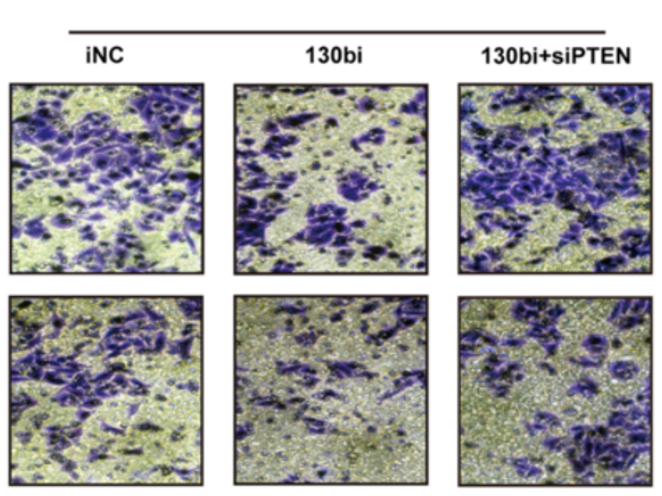

C

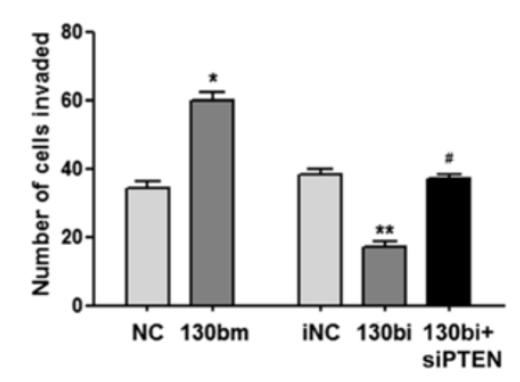

Figure 5 MiR-130b regulates the migration and invasion of ESCC cells. $1 \times 10^{5}$ Eca109 cells were added to the Transwell inserts. For the invasion assay, the wells were covered with $50 \mu \mathrm{L}$ of $1 \mathrm{mg} / \mathrm{mL}$ Matrigel. Cells were transfected with miR-130b mimic, negative control, miR-130b inhibitor, inhibitor negative control or siPTEN. The migratory or invasive cells were counted from five random areas at $200 \times$ magnification. (A) Cell images captured by microscopy. (B) Histogram of cell migration results. (C) Histogram of cell invasion results. The results are presented as mean \pm SEM from three independent experiments. ${ }^{*} P<0.05,{ }^{*} P<0.01$, ${ }^{* *} P<0.001$ vs. corresponding controls; $\# P<0.05$, \#\#P<0.01 vs. 130 bi group.

with a good prognosis. Therefore, miR-130b may play disparate tumor-associated roles that are likely related to differences in tumor types and genes targeted. To date, no published study has focused on the functions and molecular mechanisms of miR-130b in ESCC. In our study, miRNA microarray analysis and qRT-PCR confirmed that miR-130b is overexpressed in ESCC tissues. Furthermore, increased expression of miR-130b promoted proliferation, migration and invasion in vitro, whereas these abilities were weakened when miR-130b was inhibited.

PTEN is located at $10 \mathrm{q} 23.3$ and encodes a dual-specificity phosphatase with lipid and protein phosphatase activities. PTEN dephosphorylates PI $(3,4,5) \mathrm{P} 3$, an important activator of Akt [25], which controls a variety of cellular processes, such as survival, cell cycle progression, metabolism, and angiogenesis [26]. Inhibition of PTEN results in increased levels of activated p-Akt [19]. Decreased expression of PTEN in ESCC is correlated with prognosis $[27,28]$. The proliferation of ESCC cells in vitro and in vivo is promoted by transfections with PTEN expression vectors [29]. Moreover, PTEN is frequently mutated or there is loss of heterozygosity in multiple malignancies, but these occur rarely in ESCC [30,31]. Several studies have reported that certain miRNAs can directly target PTEN [25]. MiR-21 was among the earliest miRNAs for which it was confirmed that its suppression increased PTEN levels and decreased tumor cell proliferation, migration, and invasion in human hepatocellular cancer [32]. Subsequent studies indicated that PTEN is a potential target of miR-221/222, miR-22 and miR-144 in different malignancies [33-35]. Our study shows that miR-130b binds the 3'-UTR of PTEN mRNA to downregulate PTEN protein expression, thus promoting Akt phosphorylation. Moreover, silencing of PTEN expression reversed the effect of miR-130bi on cell proliferation, invasion and migration.

\section{Conclusion}

In summary, our study reveals that miR-130b is overexpressed in ESCC tissues. PTEN is a target of miR-130b, which regulates expression at the translational level. Suppression of PTEN results in increased p-Akt, promoting the proliferation, migration and invasion of ESCC cells in vitro. Our findings suggest that miR-130b may be a new target for ESCC diagnosis and therapy. However, the clinical sample size in this study was limited and the investigation of miR-130b function in ESCC was restricted to in vitro experiments. To further confirm the role of miR-130b in carcinogenesis, the expression level of miR-130b should be compared among a larger number of clinical specimens with different pathologic gradings, and also in animal models. 


\section{Additional file}

Additional file 1: The inhibition efficiency of PTEN targeted siRNA. Eca109 and TE13 cells were transfected with PTEN targeted siRNA (siPTEN) and negative control (NC) as described in the Methods section. Western blot analysis and GRT-PCR were performed to determine the protein (A) and mRNA (B) expression of PTEN. GAPDH was the endogenous control. The results are expressed as mean \pm SEM of three independent experiments. ${ }^{* *} P<0.001$ vs. corresponding controls.

\section{Abbreviations}

CCK-8: Cell counting kit-8; ESCC: Esophageal squamous cell carcinoma; GAPDH: Glyceraldehyde-3-phosphate dehydrogenase; iNC: Negative control of miRNA inhibitor; miR-130bi: miR-130b inhibitor; miR-130bm: miR-130b mimic; miRNA: microRNA; NC: Negative control of miRNA mimic; PTEN: Phosphatase and tensin homolog; p-Akt: Phosphorylated Akt; qRT-PCR: Quantitative real-time PCR; RPMI: Roswell Park Memorial Institute; SIPTEN: PTEN-targeted small interfering RNA; UTR: Untranslated region.

\section{Competing interests}

The authors declare that they have no competing interests.

\section{Authors' contributions}

TTY, RSC, QZ and RHS conceived and designed the experiments. TTY, RSC, SL, MGF, LHR and WXC performed the experiments. TTY, RSC, SL and HZ analyzed the data. TTY and RSC wrote the manuscript. All authors read and approved the final manuscript.

\section{Acknowledgements}

The study was supported by the innovation projects of Jiangsu Province Hospital (No. CX11), the National Natural Science Foundation of China (No. 81100273) and a project funded by the Priority Academic Program Development of Jiangsu Higher Education Institutions. We thank Medjaden Bioscience Limited (Hong Kong, China) and AmEditor Inc. for assisting in preparation of this manuscript.

\section{Author details}

${ }^{1}$ Department of Gastroenterology, First Affiliated Hospital of Nanjing Medical University, 300 Guangzhou Road, Nanjing 210029, China. ${ }^{2}$ Department of Gastroenterology, Wuxi People's Hospital Affiliated with Nanjing Medical University, 299 Qingyang Road, Wuxi 214023, China. ${ }^{3}$ Department of Gastroenterology, Zhongda Hospital Affiliated with Southeast University, 87 Dingjiaqiao Road, Nanjing 210009, China.

\section{Received: 17 August 2014 Accepted: 20 January 2015}

\section{Published online: 31 January 2015}

\section{References}

1. Enzinger PC, Mayer RJ. Esophageal cancer. N Engl J Med. 2003;349(23):2241-52.

2. Kamangar F, Dores GM, Anderson WF. Patterns of cancer incidence, mortality, and prevalence across five continents: defining priorities to reduce cancer disparities in different geographic regions of the world. J Clin Oncol Off J Am Soc Clin Oncol. 2006;24(14):2137-50.

3. Pennathur A, Gibson MK, Jobe BA, Luketich JD. Oesophageal carcinoma. Lancet. 2013;381(9864):400-12.

4. Toh Y, Oki E, Ohgaki K, Sakamoto Y, Ito S, Egashira A, et al. Alcohol drinking, cigarette smoking, and the development of squamous cell carcinoma of the esophagus: molecular mechanisms of carcinogenesis. Int J Clin Oncol. 2010;15(2):135-44.

5. Hongo M, Nagasaki Y, Shoji T. Epidemiology of esophageal cancer: Orient to Occident. Effects of chronology, geography and ethnicity. J Gastroenterol Hepatol. 2009;24(5):729-35.

6. Lao-Sirieix P, Caldas C, Fitzgerald RC. Genetic predisposition to gastro-oesophageal cancer. Curr Opin Genet Dev. 2010;20(3):210-7.

7. Ambros V. microRNAs: tiny regulators with great potential. Cell. 2001;107(7):823-6.

8. Calin GA, Sevignani C, Dumitru CD, Hyslop T, Noch E, Yendamuri S, et al. Human microRNA genes are frequently located at fragile sites and genomic regions involved in cancers. Proc Natl Acad Sci U S A. 2004;101(9):2999-3004.
9. Nana-Sinkam SP, Croce CM. Clinical applications for microRNAs in cancer Clin Pharmacol Ther. 2013;93(1):98-104.

10. Lu J, Getz G, Miska EA, Alvarez-Saavedra E, Lamb J, Peck D, et al. MicroRNA expression profiles classify human cancers. Nature. 2005;435(7043):834-8.

11. Ferracin M, Veronese A, Negrini M. Micromarkers: miRNAs in cancer diagnosis and prognosis. Expert Rev Mol Diagn. 2010;10(3):297-308.

12. Burmistrova OA, Goltsov AY, Abramova LI, Kaleda VG, Orlova VA, Rogaev El. MicroRNA in schizophrenia: genetic and expression analysis of miR-130b (22q11). Biochemistry Biokhimiia. 2007;72(5):578-82.

13. Lai KW, Koh KX, Loh M, Tada K, Subramaniam MM, Lim XY, et al. MicroRNA-130b regulates the tumour suppressor RUNX3 in gastric cancer. Eur J Cancer. 2010;46(8):1456-63.

14. Li BL, Lu C, Lu W, Yang TT, Qu J, Hong X, et al. miR-130b is an EMT-related microRNA that targets DICER1 for aggression in endometrial cancer. Med Oncol. 2013;30(1):484.

15. Ma S, Tang KH, Chan YP, Lee TK, Kwan PS, Castilho A, et al. miR-130b Promotes CD133(+) liver tumor-initiating cell growth and self-renewal via tumor protein 53-induced nuclear protein 1. Cell Stem Cell. 2010;7(6):694-707.

16. Yang C, Cai J, Wang Q, Tang H, Cao J, Wu L, et al. Epigenetic silencing of miR-130b in ovarian cancer promotes the development of multidrug resistance by targeting colony-stimulating factor 1. Gynecol Oncol. 2012;124(2):325-34.

17. Yip L, Kelly L, Shuai Y, Armstrong MJ, Nikiforov YE, Carty SE, et al. MicroRNA signature distinguishes the degree of aggressiveness of papillary thyroid carcinoma. Ann Surg Oncol. 2011;18(7):2035-41.

18. Zhao BS, Liu SG, Wang TY, Ji YH, Qi B, Tao YP, et al. Screening of microRNA in patients with esophageal cancer at same tumor node metastasis stage with different prognoses. Asian Pac J Cancer Prev. 2013;14(1):139-43.

19. Xu M, Mo YY. The Akt-associated microRNAs. Cell Mol Life Sci. 2012;69(21):3601-12.

20. Friedman RC, Farh KK, Burge CB, Bartel DP. Most mammalian mRNAs are conserved targets of microRNAs. Genome Res. 2009;19(1):92-105.

21. Hobert $O$. Gene regulation by transcription factors and microRNAs. Science. 2008;319(5871):1785-6.

22. Lewis BP, Burge CB, Bartel DP. Conserved seed pairing, often flanked by adenosines, indicates that thousands of human genes are microRNA targets. Cell. 2005;120(1):15-20.

23. Esquela-Kerscher A, Slack FJ. Oncomirs - microRNAs with a role in cancer. Nat Rev Cancer. 2006;6(4):259-69.

24. Bandyopadhyay S, Mitra R, Maulik U, Zhang MQ. Development of the human cancer microRNA network. Silence. 2010;1(1):6.

25. Song MS, Salmena L, Pandolfi PP. The functions and regulation of the PTEN tumour suppressor. Nat Rev Mol Cell Biol. 2012;13(5):283-96.

26. Blanco-Aparicio C, Renner O, Leal JF, Carnero A. PTEN, more than the AKT pathway. Carcinogenesis. 2007;28(7):1379-86.

27. Chang D, Wang TY, Li HC, Wei JC, Song JX. Prognostic significance of PTEN expression in esophageal squamous cell carcinoma from Linzhou City, a high incidence area of northern China. Dis Esophagus. 2007;20(6):491-6.

28. Tachibana M, Shibakita M, Ohno S, Kinugasa S, Yoshimura H, Ueda S, et al. Expression and prognostic significance of PTEN product protein in patients with esophageal squamous cell carcinoma. Cancer. 2002;94(7):1955-60.

29. Zhou YA, Zhang T, Zhao JB, Wang XP, Jiang T, Gu ZP, et al. The adenovirusmediated transfer of PTEN inhibits the growth of esophageal cancer cells in vitro and in vivo. Biosci Biotechnol Biochem. 2010;74(4):736-40.

30. Ding Y, Shimada Y, Kano M, Itami A, Kawabe A, Maeda M, et al. PTEN/MMAC1 expression in esophageal squamous cell carcinomas. Int J Oncol. 2000;17(4):695-9.

31. Hu YC, Lam KY, Tang JC, Srivastava G. Mutational analysis of the PTEN/ MMAC1 gene in primary oesophageal squamous cell carcinomas. Mol Pathol. 1999;52(6):353-6.

32. Meng F, Henson R, Wehbe-Janek H, Ghoshal K, Jacob ST, Patel T. MicroRNA-21 regulates expression of the PTEN tumor suppressor gene in human hepatocellular cancer. Gastroenterology. 2007;133(2):647-58.

33. Bar N, Dikstein R. miR-22 forms a regulatory loop in PTEN/AKT pathway and modulates signaling kinetics. PLoS One. 2010;5(5):e10859.

34. Garofalo M, Di Leva G, Romano G, Nuovo G, Suh SS, Ngankeu A, et al. miR-221\&222 regulate TRAIL resistance and enhance tumorigenicity through PTEN and TIMP3 downregulation. Cancer Cell. 2009;16(6):498-509.

35. Zhang LY, Ho-Fun Lee V, Wong AM, Kwong DL, Zhu YH, Dong SS, et al. MicroRNA-144 promotes cell proliferation, migration and invasion in nasopharyngeal carcinoma through repression of PTEN. Carcinogenesis. 2013;34(2):454-63. 